\title{
Life events, emotional responsiveness, and the functional prognosis of patients with rheumatoid arthritis
}

\author{
Jun Nagano ${ }^{*}$, Nobuyuki Sudo ${ }^{2}$, Shohei Nagaoka ${ }^{3}$, Masao Yukioka ${ }^{4}$ and Masakazu Kondo ${ }^{5}$
}

\begin{abstract}
Background: Stressors may differently affect human physiological systems according to the host properties relevant to psycho-behavioral processes that the stressors invoke. In a Japanese multicenter cohort study of patients with rheumatoid arthritis (RA), we examined if major life events differently contribute to the patients' functional prognosis according to their ability to identify emotions as manifest feelings when encountering the events (emotional responsiveness).

Methods: 460 patients with RA completed a self-administered baseline questionnaire about psychosocial factors including emotional responsiveness. Two years later, they checked on a list of positive/negative personal events that happened during the two-year study period. Rheumatologists evaluated their functional status at baseline and follow-up using the ACR classification system.

Results: In a multiple logistic regression model that included baseline demographic, disease activity/severity-related, therapeutic, and socioeconomic factors as covariates, none of the counts of positive, negative, or all life events was associated with the functional status at follow-up. In the subgroup with poor emotional responsiveness, however, these life event counts were all associated with a poorer functional prognosis (odds ratio of ACR class 3-4 vs. 1-2 associated with one increment in the all life-event count $=2.39,95 \%$ confidence interval $=1.27-4.48, p=.007$ ), while no such relationship was evident for the rest of the patients.
\end{abstract}

Conclusions: Major life events, whether positive or negative in nature, may have an impact on the disease course of patients with RA when the patient has poor emotional responsiveness to the event(s).

Keywords: Arthritis, Rheumatoid, Stress, Psychological, Personality, Emotions, Function, Prospective studies

\section{Background}

Psychological stress (stress) is determined not only by external stressors, but also by interactions with internal conditions, i.e., emotional, cognitive, and behavioral response properties [1]. Thus, it is important to take individual response properties into consideration when addressing the effects of external events that place stress on the organism. Major life-event type stressors alone have not been observed to precede the onset of rheumatoid arthritis (RA) [2], an autoimmune disease characterized by chronic systemic inflammation that mainly affects joints leading to a loss of physical functioning [3],

\footnotetext{
* Correspondence: jun@artsci.kyushu-u.ac.jp

${ }^{1}$ Faculty of Arts and Science, Kyushu University, 6-1 Kasuga Park, Kasuga,

Fukuoka 816-8580, Japan

Full list of author information is available at the end of the article
}

although the psychosomatic aspects of RA have been considered for decades [4, 5]. Moreover, life events have been suggested to be associated with a decrease in the disease activity of RA [6]. As regards individual properties (reaction to stressors), however, patients with RA who are easily moved to tears as a response to psychological stress have been reported to show a better response to treatment and a better general prognosis than those who did not show such an emotional response [7]. In addition, we recently reported that "rational and antiemotional" behavior (antiemotionality), characterized by an extreme tendency to suppress emotional behaviors and to rationalize negative experiences in conflicting interpersonal situations, is associated with a poor functional prognosis for patients with RA [8].
Ciomed Central

(c) 2015 Nagano et al. This is an Open Access article distributed under the terms of the Creative Commons Attribution License (http://creativecommons.org/licenses/by/4.0), which permits unrestricted use, distribution, and reproduction in any medium, provided the original work is properly credited. The Creative Commons Public Domain Dedication waiver (http:// creativecommons.org/publicdomain/zero/1.0/) applies to the data made available in this article, unless otherwise stated. 
In order to invoke such cognitive and behavioral responses as expression/suppression of emotions, however, life events must arouse emotions that patients are aware of and that they identify as feelings, i.e., subjective and conscious experiences $[1,9,10]$. Persons who lack strong, positive or negative emotional experiences in life may have diminished ability to detect and identify emotions or have relevant processes that are strongly suppressed. When emotions are inappropriately processed, the limbic system, the center of emotions, might interfere with the homeostasis of the organism, including the immune system, through the hypothalamus-pituitary-adrenal (HPA) axis and the hypothalamus-autonomic nervous system (ANS), thereby affecting the functional prognosis of patients with RA $[6,11,12]$. We tested these hypotheses by calculating the self-reported major life events that occurred within a given period, two years, with a lifelong lack of emotional experiences as an index for poor detection/identification of emotions, antiemotionality as a poor coping behavior, and the transition of physical functional status as an organic change, on the basis of data from a cohort study of Japanese RA patients.

\section{Methods}

\section{Subjects}

The subjects were patients who participated in the Assessment and Improvement of the System for Interdisciplinary Medical Services for RA (AISIMS) cohort study [13] and whose follow-up data two years after the baseline survey was available. The AISIMS cohort study is a multi-center survey of Japanese patients with RA that started in 2000 with support from the Ministry of Health and Welfare of Japan. Twelve hospitals/clinics located across Japan participated in the study program, of which eight cooperated in the follow-up survey. A series of 532 eligible patients with RA who were regularly visiting one of the eight hospitals/ clinics and who met the following criteria completed a self-administered questionnaire for the baseline survey in 2000. The survey inquired about a variety of factors, including activity of daily living, quality of life, lifestyle, and psychosocial factors (major life events, stress/personality, etc.). Eligibility criteria were age between 20 and 79 years, the ability to answer a self-administered questionnaire without assistance, and functional status of class III or lower according to the American College of Rheumatology (ACR) (see below) [14]. A total of 23 rheumatologists responsible for the patients completed a baseline clinical data sheet that included information about the disease status, such as the progression of arthritis, functional status, extra-articular complications, and medical treatment. The same rheumatologists again completed the clinical data sheet for each of the 479 patients who continued visiting to the time of the follow-up survey, which included a questionnaire with items selected from the baseline questionnaire. The 460 cooperating patients (406 women, mean age 56.1 years) mailed their completed questionnaires to a central office (see Fig. 1). The rheumatologists who completed the clinical data sheets were blinded to the patients' answers for both the baseline and follow-up questionnaires. A more detailed description of the subjects has been reported elsewhere [8].

\section{Measurements}

The rheumatologists assessed their patients' functional status based on the criteria for the classification of functional status of RA patients defined by ACR (ACR class), which classifies the patients into one of four classes as follows: Class I, completely able to perform usual activities of daily living (self-care, vocational, and avocational); Class II, able to perform usual self-care and vocational activities, but limited in avocational activities; Class III, able to perform usual self-care activities, but limited in vocational and avocational activities; and Class IV, limited in ability to perform usual self-care, vocational, and avocational activities [14]. They also assessed joint damage based on the classification by Steinbrocker et al. (joint stage: Stage I, Early; Stage II, Moderate; Stage III, Severe; and Stage IV, Terminal) [15]; specified afflicted joints defined as those with either tenderness, swelling, or deformity; and listed extra-articular complications using the following options: cervical myelopathy, cardiac/pericardial manifestations, pulmonary/pleural manifestations, ocular manifestations, peripheral nervous manifestations, hematological manifestations, and others.

Major life events that occurred in the two-year follow-up period were assessed using a self-administered questionnaire that was developed specifically for the ASIMS cohort study. It consists of an instruction sentence, "please answer if any of the following eight major life events happened to you in the past two years": 1. Got married; 2 . Went through bereavement or parted with an important person; 3. I or my partner got a new job; 4. I or my partner lost a job (excluding retirement); 5 . Financially became much better off than before; 6 . Financially got into a serious situation; 7. A major family problem was resolved; and 8. A major family problem occurred. Items $1,3,5$, and 7 are positive events, and items $2,4,6$, and 8 are negative events. These items were prepared referring to reports that graded major life events according to the possible magnitude at which the events would impact life [16]; the grading is very similar between the original survey in the U.S.A. and replication studies done in Japan [17, 18]. The subjects ticked either "happened" or "did not happen" for each item. The same questionnaire, with one difference in that the instruction sentence referred to life events that happened in the previous year, was also included in the baseline survey to attempt to cross-validate it with an instrument that measures emotional responsiveness to major 


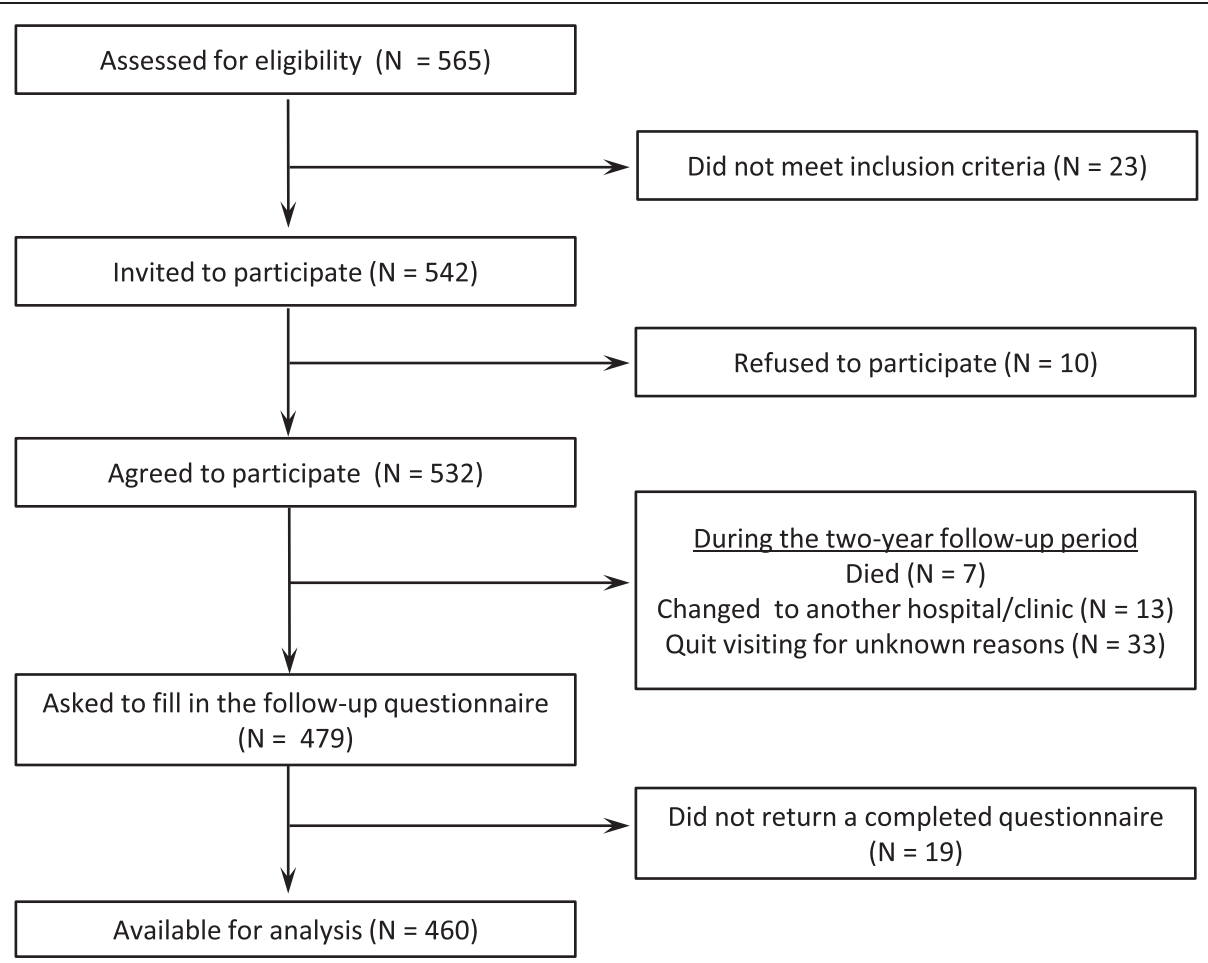

Fig. 1 Flow diagram

life events (see below). This issue is discussed in the Discussion section of this paper.

The psycho-behavioral properties detection and identification of emotions and coping behaviors were assessed using the "Stress Inventory (SI)" [19, 20]. The $\mathrm{SI}$ is a 45 -item self-administered questionnaire that was developed to assess response styles to stressors principally related to an interpersonal relationship or to chronic stress posed by the response style. Of the 12 scales constituting the SI, the "lack of emotional experiences (LEE)" scale attempts to assess poor emotional responsiveness to major life events, i.e., diminished ability to detect and identify strong emotions as manifest feelings when a person encounters positive and negative major life events. This scale consists of four questions, such as "in your whole life, have you experienced outrage about something?" and "in your whole life, have you experienced jumping for joy about something?" (Cronbach $\alpha=0.60)$. The "rationalizing conflicts/frustrations (RCF)" scale was developed to represent antiemotionality. It measures an extreme tendency to rationalize one's interpersonal situations accompanied by conflicts or frustrations and it consists of five questions, such as "do you under all circumstances try to control your reasoning and avoid, as much as possible, being emotional?" (Cronbach $\alpha=0.78$ ). The answers receive a 1 to 6 rating, where 1 and 6 respectively correspond to "yes" and "no", and the scores are averaged for the scale score, thus a higher score represents a higher tendency. While the concept of antiemotionality represents a behavioral response style in daily situations that distinctly arouse conflicting or frustrating emotions, the core concept of poor emotional responsiveness represents a more biological, rather than behavioral, response style when encountering major life events [19]. In a psychometrical validation study, for example, while RCF was moderately correlated with anger traits, LEE was only weakly correlated, if any [20]. The RCF and LEE scores in the present study were statistically independent (Spearman's rank correlation coefficient $=-0.03$ ).

\section{Analysis}

The ACR class at follow-up was dichotomized (poorer function, Class III or IV vs. better function, Class I or II) for use as the outcome variable representing the functional prognosis of the patients with RA. "Happened" items in the life event questionnaire were counted separately for positive (0-4) and negative events (0-4), and also aggregated for all events (0-8). Thus for example, if a patient ticked two positive event "happened" boxes and one negative event "happened" boxes, the life event counts for the patient would be 2, 1, and 3 for positive, negative, and all events, respectively. The associations between these life event counts and the functional prognosis were examined using a multiple logistic regression model that included one of these life event counts as an independent variable, the dichotomized ACR class at 
follow-up (better function $=0$, poorer function $=1$ ) as the dependent variable, and baseline ACR class (Class I, II, III, IV $=1,2,3,4$, respectively) as a covariate. The models also included known or potential confounding factors relevant to disease progression or activity: joint stage (Stage I, II, III, IV = 1, 2, 3, 4, respectively), afflicted joints count (1-45), number of extra-articular complications (0-5), C-reactive protein with log-transformation; medical treatments: methotrexate, corticosteroids, other DMARDs (yes $=1$, no $=0$ ); and socioeconomic status: education level (junior high $\mathrm{school}=1$, high $\operatorname{school}=2$, junior college $=3$, college $=4$ ). These analyses were then applied to the subgroups divided based on either the degree of the LEE score or that of the RCF score, using the median of each score as the cut-point. High $(>=2.5$ points) and low ( $<2.5$ points) LEE scores represent high and low degrees of poor emotional responsiveness; and high ( $>=4.4$ points) and low ( $<4.4$ points) RCF scores represent high and low degrees of antiemotionality. The reported $p$-values are two-sided, and values $<.05$ were considered statistically significant. All analyses were done using SAS v. 9.2.

\section{Results}

Table 1 shows the baseline characteristics of the patients according to their level of emotional responsiveness. Poorer emotional responsiveness was associated with male sex, older age, lower education level, more advanced joint stage,

Table 1 Baseline association of poor emotional responsiveness with the demographic and clinical characteristics of patients with rheumatoid arthritis

\begin{tabular}{llll}
\hline & \multicolumn{2}{l}{$\begin{array}{l}\text { Poor emotional } \\
\text { responsiveness }\end{array}$} & $p^{\mathrm{b}}$ \\
& Low & High & \\
& $N=252$ & $N=208$ & \\
\hline Female & 91.7 & 84.1 & .013 \\
Age, yrs, mean (SD) & $54.3(9.7)$ & $58.4(9.0)$ & $<.001$ \\
Education, college or higher & 24.2 & 16.4 & .038 \\
Duration, yrs, mean (SD) & $11.6(9.7)$ & $11.1(9.3)$ & .58 \\
ACR class > =3 & 13.5 & 13.5 & .99 \\
Joint stage > =3 & 62.7 & 71.6 & .043 \\
Afflicted joints count, mean (SD) & $9.4(8.2)$ & $10.6(9.4)$ & .15 \\
No. extra-articular complications $>=1$ & 22.6 & 25.0 & .55 \\
CRP, mg/dl, mean (SD) & $1.42(1.82)$ & $1.88(2.40)$ & .019 \\
Methotrexate use & 42.1 & 42.3 & .96 \\
Corticosteroids use & 50.4 & 37.5 & .006 \\
Other DMARDs use & 55.2 & 51.4 & .43 \\
\hline
\end{tabular}

Values are \% unless otherwise stated. ACR: American College of Rheumatology, CRP: C-reactive protein, DMARDs: disease modifying antirheumatic drugs. ${ }^{a}$ The SI "lack of emotional experiences" score $(19,20)$ : low score $<2.5$ points, high score $>=2.5$ points. 'Based on Spearman's rank correlation higher CRP, and lower corticosteroid use. Greater antiemotionality was associated with female sex, older age, and greater number of afflicted joints: The corresponding table has been presented elsewhere [8]. Table 2 shows the number of subjects who reported having experienced a life event during the two-year observation period. The most frequent event was a bereavement/parting with an important person, followed by a financial crisis, with over $15 \%$ of the patients having experienced one of these negative events. When the events were totaled, the number of patients who reported one event and two or more events were respectively 71 $(15.5 \%)$ and $23(5.0 \%)$ for positive events, $120(26.1 \%)$ and $58(12.6 \%)$ for negative events, and $126(27.5 \%)$ and 93 (20.3\%) for all events.

The distribution of ACR class changed from baseline to the follow-up as follows: Class 1, 76 (16.5\%) to 65 (14.2\%); Class 2, 322 (70.0 \%) to 327 (71.2 \%); Class 3, 62 (13.5 \%) to $58(12.6 \%)$; and Class 4, $0(0 \%)$ to $9(2.0 \%)$. The global functional status had improved for $56(12.2 \%)$ patients, was unchanged for 327 (71.2\%), and had deteriorated for 76 (16.6\%) over the two-year study period. In addition to the ACR class at baseline, the afflicted joints count and steroid use were significantly associated and extra-articular complication was marginally significantly associated with the functional prognosis in a multiple logistic regression model that included the baseline characteristics as independent variables (data not shown). In the multiple logistic regression model that included these baseline factors as covariates, emotional responsiveness was not associated with the functional prognosis (OR associated with one-point increment in the LEE score $=0.97,95 \%$ $\mathrm{CI}=0.71-1.33, p=.83$ ). The positive (OR associated with one-event increment $=1.20,95 \% \mathrm{CI}=0.68-2.13$, $p=.53)$, negative $(\mathrm{OR}=1.25, \mathrm{CI}=0.81-1.94, p=.32)$, and all life event counts $(\mathrm{OR}=1.31, \mathrm{CI}=0.88-1.94, p=.18)$

Table 2 Positive and negative life events reported to have occurred in the lives of patients with rheumatoid arthritis during the two-year study period

\begin{tabular}{|c|c|c|}
\hline & Number $^{a}$ & Percent $^{b}$ \\
\hline \multicolumn{3}{|l|}{ Positive events } \\
\hline 1. ${ }^{c}$ Got married. & 10 & 2.2 \\
\hline 3. I or my partner got a new job. & 28 & 6.1 \\
\hline 5. Financially became much better off than before. & 41 & 8.9 \\
\hline 7. A major problem within my family was resolved. & 28 & 6.1 \\
\hline \multicolumn{3}{|l|}{ Negative events } \\
\hline $\begin{array}{l}\text { 2. Bereavement or parting from an important } \\
\text { person. }\end{array}$ & 73 & 15.9 \\
\hline 4. I or my partner lost a job (excluding retirement). & 30 & 6.5 \\
\hline 6. Financially got into a serious situation. & 71 & 15.4 \\
\hline 8. A major problem came about in my family. & 53 & 11.5 \\
\hline
\end{tabular}

aNumber of patients who ticked the "happened" box that corresponds to the event, and ${ }^{\mathrm{b}}$ proportion [number/total $\left.(N=460)\right]$. ${ }^{\mathrm{C}} \mathrm{Question}$ number 
were not significantly associated with the functional prognosis in the analysis of all patients.

As shown in Table 3, however, when the patients were divided into two subgroups by the degree of emotional responsiveness (LEE score), the associations of life events were different. For the patients whose emotional responsiveness was not poor the associations were null or inverse, if any, while for those with poor emotional responsiveness positive, negative, and all events were all significantly associated with poorer functional status at follow-up. Thus, for patients with poor emotional responsiveness, experiencing a major life event was associated with a two or greater fold chance of having a poorer functional status in the subsequent two years. Such a difference in the events/prognosis associations was not evident when the data was stratified by the dichotomized level of antiemotionality (RCF score) (Table 3 ). Figure 2 shows the transition of ACR class associated with the number of major life events in the study period, according to the degree of emotional responsiveness. In the subgroup with poor emotional responsiveness (right), the percentage of patients whose functional class deteriorated in the study period tended to be higher as the number of life events increased: $10.3 \%$ (12 of 116), $17.4 \%$ (8 of 46), and $23.9 \%$ (11 of 46 ) for those who reported none, one, and two or more events, respectively. In the subgroup with better emotional responsiveness (left), however, such tendency was not evident, with the corresponding percentages $20.8 \%$ (26 of 125 ), $15.0 \%$ (12 of 80 ), and $14.9 \%$ (7 of $47)$, respectively.

\section{Discussion}

This Japanese, multicenter, cohort study of patients with RA found that a lack of experiences accompanied by strong feelings caused by positive and negative life events was associated with a poor functional prognosis. This is, to the authors' knowledge, the first epidemiological study to report the possibility that life-event type stressors impact the disease course of RA patients according to their ability to detect and identify their emotions.

The death of a child as a major life event has been reported to not increase the risk of onset (new admission) of RA of the parents [2]. This is in line with the present finding that life events were not associated with the functional prognosis of these RA patients when the data is taken as a whole. However, when the data is limited to patients who were thought to have poor emotional responsiveness as an antecedent condition, life events, irrespective of their positive or negative nature, were associated with their functional prognosis.

The four items of the LEE scale asked the patients if they had "not at all" experienced an incident accompanied by a strong positive or negative emotional response. Of the patients, 77 basically agreed to these questions ( 3.5 points or higher on average), but $18 \%, 38 \%$, and $43 \%$ reported having experienced one or more positive, negative, or any life event, respectively, during the year previous to the baseline survey. Moreover, these proportions were similar for the other 383 patients ( $20 \%, 35 \%$, and $46 \%$, respectively). Thus, it would be more natural to think that even major events have minimally aroused emotional responses that those who relatively favored the LEE questions are manifestly aware of, rather than that no major event had actually happened in their life. When events arouse well-identified feelings, a person would initiate coping behaviors, thereby alleviating the events' potential health effects [10]. On the other hand, external stimuli, when they were not sensed, may affect the immune system through the HPA axis and the hypothalamus/ANS, which are closely linked with the immune system and thereby impact the functional prognosis of RA patients.

Table 3 Multiple logistic regression analysis of the association between life events that occurred during the two-year study period and the functional prognosis of patients with rheumatoid arthritis, stratified by dichotomized levels of the baseline behavioral patterns

\begin{tabular}{|c|c|c|c|c|c|c|c|c|c|c|c|c|}
\hline \multirow[t]{6}{*}{ Life events } & \multicolumn{12}{|c|}{ Baseline behavioral pattern } \\
\hline & \multirow{2}{*}{\multicolumn{6}{|c|}{$\begin{array}{l}\text { Poor emotional responsiveness } \\
(\text { LEE score })^{a}\end{array}$}} & \multirow{2}{*}{\multicolumn{6}{|c|}{$\begin{array}{l}\text { Antiemotionality } \\
(\text { RCF score })^{b}\end{array}$}} \\
\hline & & & & & & & & & & & & \\
\hline & \multirow{2}{*}{\multicolumn{3}{|c|}{$\begin{array}{l}\text { Low } \\
N=252\end{array}$}} & \multirow{2}{*}{\multicolumn{3}{|c|}{$\begin{array}{l}\text { High } \\
N=208\end{array}$}} & \multirow{2}{*}{\multicolumn{3}{|c|}{$\begin{array}{l}\text { Low } \\
N=217\end{array}$}} & \multirow{2}{*}{\multicolumn{3}{|c|}{$\begin{array}{l}\text { High } \\
N=242\end{array}$}} \\
\hline & & & & & & & & & & & & \\
\hline & $\mathrm{OR}$ & $(95 \% \mathrm{Cl})^{\mathrm{C}}$ & $p$ & $\overline{\mathrm{OR}}$ & $(95 \% \mathrm{Cl})$ & $p$ & $\mathrm{OR}$ & $(95 \% \mathrm{Cl})$ & $p$ & $\overline{\mathrm{OR}}$ & $(95 \% \mathrm{Cl})$ & $p$ \\
\hline Positive events & 0.60 & $(0.23-1.53)$ & .28 & 2.73 & $(1.13-6.58)$ & .026 & 0.82 & $(0.27-2.48)$ & .73 & 1.46 & $(0.69-3.11)$ & .32 \\
\hline Negative events & 0.82 & $(0.42-1.63)$ & .58 & 1.99 & $(1.03-3.86)$ & .041 & 1.87 & $(0.93-3.75)$ & .08 & 0.81 & $(0.42-1.56)$ & .53 \\
\hline All events & 0.77 & $(0.42-1.40)$ & .39 & 2.39 & $(1.27-4.48)$ & .007 & 1.59 & $(0.83-3.04)$ & .16 & 1.11 & $(0.64-1.94)$ & .71 \\
\hline
\end{tabular}

OR: odds ratio. Cl: confidence interval. ACR: American College of Rheumatology. DMARDs: disease modifying anti-rheumatic drugs. ${ }^{2}$ The lack of emotional experiences (LEE) score of the Stress Inventory (19,20): low score $<2.5$ points (median), high score $>=2.5$ points. ${ }^{\text {b}}$ The rationalizing conflicts/frustrations (RCF) score of the Stress Inventory: low score $<4.4$ points (median), high score $>=4.4$ points. ${ }^{\mathrm{C} O R}$ of poorer (ACR class at follow-up $>=3$ ) vs. better (ACR class at follow-up $<=2$ ) functional prognosis associated with one-point increment in the number of life events experienced in the two-year study period, controlled for age, sex, education level, and baseline measurements including ACR class, C-reactive protein, extra-articular complications, methotrexate, corticosteroids, and other DMARDs 

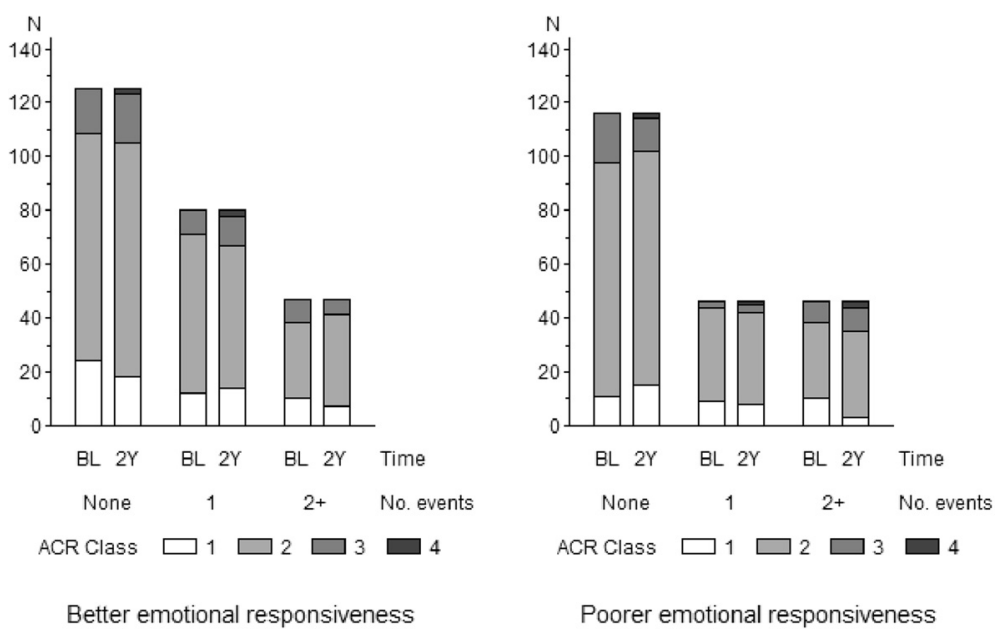

Fig. 2 The association of the number of major life events experienced by patients with rheumatoid arthritis in the two-year study period and the transition of their physical functional status according to their degree of emotional responsiveness. N, number of subjects; BL, at the time of the baseline survey; $2 Y$, two years after the baseline survey. No. events: the number of life events reported to have happened in the two-year study period. ACR: American College of Rheumatology. Better and poorer emotional responsiveness are represented by low ( $<2.5$ points) and high $(2.5+$ points) scores for the lack of emotional experiences scale from the Stress Inventory $(19,20)$

The LEE score was associated with several baseline factors, especially higher age and regular use of corticosteroids. Aging and steroid use may contribute to the functioning of emotional responsiveness as assessed using the score, but the present data lacks information that would be helpful for further understanding this issue. However, the association between life events and the ACR class at follow-up in the subgroup with a high LEE score was evident in the model that included age and steroid use as covariates, thus the association would be independent of age and steroid use.

Although antiemotionality was associated with a poor functional prognosis for patients with RA in the present cohort [8], it was not apparent that this behavioral property modified the effects of major life events on the health outcome. Major and minor stressors have been reported to be different in the nature of the immunological response to them by patients with RA $[6,21]$. Antiemotionality is a behavioral pattern in response to detected/identified unpleasant emotions. A personality characteristic that overly rationalizes and suppresses sensed feelings would, when such psychological processes are repeated in response to minor daily events, affect the physiological processes, whereas it might have a positive aspect as a coping behavior to a major event, i.e., when strong unpleasant feelings are aroused by the major event, antiemotionality may sometimes be beneficial in realistically dealing with the problems related to the event, which might cancel out the property's negative aspects.

Several limitations of the study need to be discussed. First, no biomarkers, such as neuroendocrinological or immunological markers were measured, and the underlying pathways that connect emotions to physiological changes are largely unknown. Second, the outcome measure used, ACR class, is limited in its sensitivity in detecting temporal changes of the status [22], and the assessment depends completely on the subjective evaluation of the rheumatologist. Third, a possible recall bias should be considered, because the assessment of life events depended on the subjects' ability to remember events that happened over the two years before the follow-up. Thus, a person whose emotional responsiveness is diminished may underestimate their life events. Such a bias, however, may not be a major concern because the LEE score was not correlated with the reported number of life events that occurred in the previous year (Spearman's rank correlation coefficients were $-0.04,-0.06$, and -0.07 respectively for positive, negative and all life events in the present data). Fourth, the scale used to assess life events was a simple tool that consists of only eight items (see Table 3), thus we could not further discuss the effects of more concrete and more diverse events. The LEE scale is also a simple tool that consists of only four items; it is thought to share its construct to some extent with a component of alexithymia, a personality construct characterized by the sub-clinical inability to identify and describe one's own emotions [23, 24] which has been linked to physical health problems [25, 26], but this issue has not yet been examined. On the other hand, the simplicity of these tools would simplify replication studies with larger samples or that are done in a daily clinical practice setting. Further attempts to validate the construct that the LEE measures via correlation analyses with relevant 
psychometrical tools such as the Toronto Alexithymia Scale [27], the Levels of Emotional Awareness Scale [28], and/or with neuroimaging techniques [29] would be useful.

\section{Conclusions}

Major life events, irrespective of their positive or negative nature, were associated with the disease course of patients with RA when the patient has poor ability in identifying emotions as manifest feelings (emotional responsiveness). Thus, emotional responsiveness may be an important contributing factor to the physiological and organic responses that follow major events in the life of patients with RA.

\begin{abstract}
Abbreviations
ACR: American College of Rheumatology; AISIMS: Assessment and improvement of the system for interdisciplinary medical services for rheumatoid arthritis; ANS: Autonomic nervous system;

Antiemotionality: Rational and antiemotional behavior; DMARD:

Disease-modifying antirheumatic drug; HPA: Hypothalamus-pituitary-adrenal; RA: Rheumatoid arthritis; RCF: Rationalizing conflicts/frustrations; SI: the Stress inventory.

\section{Competing interests}

The authors declare that they have no competing interests.

\section{Authors' contributions}

JN conceived of the study. JN, SN, MY, and MK designed the study and collected the data. JN and NS analyzed the data. JN prepared the draft of the manuscript and NS helped draft the manuscript. All authors read and approved the final manuscript.
\end{abstract}

\section{Acknowledgments}

The hospitals/clinics that participated in the present cohort study were the Japanese Red Cross Kitami Hospital (Hokkaido prefecture), Yokohama Minami Kyousai Hospital (Kanagawa), Hot Spring of Rehabilitation Nakaizu Hospital (Shizuoka), National Nagoya Hospital (Aichi), Yukioka Hospital (Osaka), Miki Sanyo Hospital (Hyogo), Dohgo Spa Hospital (Ehime), and the Kondo Rheumatism and Orthopedics Clinic (Fukuoka). The authors thank Takako Morita, Koji Taneichi, Sadanobu Katsube, Tomiaki Asai, Kiyoshi Takasugi and Yasuro Nishibayashi for assisting them with the study design and data collection. This work was supported by a Health Sciences Research Grant from the Ministry of Health and Welfare of Japan and a Grant-in-Aid for Scientific Research (21590765) from the Ministry of Education, Culture, Sports, Science and Technology of Japan.

\section{Author details \\ ${ }^{1}$ Faculty of Arts and Science, Kyushu University, 6-1 Kasuga Park, Kasuga, Fukuoka 816-8580, Japan. ${ }^{2}$ Department of Psychosomatic Medicine, Kyushu University Graduate School of Medical Sciences, 3-1-1 Maidashi, Higashi-ku, Fukuoka 812-8582, Japan. ${ }^{3}$ Yokohama Minami Kyousai Hospital, 1-21-1 Mutsu-ura Higashi Kanazawa-ku, Yokohama 236-0037, Japan. ${ }^{4}$ Yukioka Hospita, 2-2-3 Ukida, Kita-ku, Osaka 530-0021, Japan. ${ }^{5}$ Kondo Rheumatism and Orthopedics Clinic, 3-10-11 Tenjin, Chuo-ku, Fukuoka 810-0000, Japan.}

Received: 19 March 2015 Accepted: 19 June 2015

Published online: 23 June 2015

\section{References}

1. Lazarus RS. Coping theory and research: past, present, and future. Psychosom Med. 1993;55:234-47.

2. Li J, Schiottz-Christensen B, Olsen J. Psychological stress and rheumatoid arthritis in parents after death of a child: a national follow-up study. Scand J Rheumatol. 2005;34:448-50.

3. Scott DL, Wolfe F, Huizinga TW. Rheumatoid arthritis. Lancet. 2010;376:1094-108

4. Alexander F. Psychosomatic Medicine. Its Principles and Applications. New York: W. W. Norton \& Co. Press; 1950.

5. Stojanovich L, Marisavljevich D. Stress as a trigger of autoimmune disease. Autoimmun Rev. 2008;7:209-13.
6. Cutolo M, Straub RH. Stress as a risk factor in the pathogenesis of rheumatoid arthritis. Neuroimmunomodulation. 2006;13:277-82.

7. Ishii H, Nagashima M, Tanno M, Nakajima A, Yoshino S. Does being easily moved to tears as a response to psychological stress reflect response to treatment and the general prognosis in patients with rheumatoid arthritis? Clin Exp Rheumatol. 2003;21:611-6.

8. Nagano J, Morita T, Taneichi K, Nagaoka S, Katsube S, Asai T, Yukioka M, Takasugi K, Kondo M, Nishibayashi Y. Rational/antiemotional behaviors in interpersonal relationships and the functional prognosis of patients with rheumatoid arthritis: a Japanese multicenter, longitudinal study. BioPsychoSocial Medicine. 2014;8:8.

9. Lane RD, Schwartz GE. Levels of emotional awareness: a cognitivedevelopmental theory and its application to psychopathology. Am J Psychiatry. 1987;144:133-43.

10. Subic-Wrana C, Beutel ME, Brahler E, Stobel-Richter Y, Knebel A, Lane RD, Wiltink J. How is emotional awareness related to emotion regulation strategies and self-reported negative affect in the general population? PLoS ONE. 2014;9:e91846.

11. Jessop DS, Richards $\sqcup$, Harbuz MS. Effects of stress on inflammatory autoimmune disease: destructive or protective? Stress. 2004;7:261-6.

12. de Brouwer SJ, Kraaimaat FW, Sweep FC, Creemers MC, Radstake TR, van Laarhoven Al, van Riel PL, Evers AW. Experimental stress in inflammatory rheumatic diseases: a review of psychophysiological stress responses. Arthritis Res Ther. 2010;12:R89.

13. Nishibayashi Y. Assessment and improvement of the system for interdisciplinary medical service for rheumatoid arthritis. In: Kudo H, editor. Report for Research on Immunological and Allergic disease, Health Sciences Research Grant 2001. Volume 1. Tokyo: Bureau of the Research on Immunological and Allergic disease, Japan Ministry of Health and Welfare; 2002. p. 1-9.

14. Hochberg MC, Chang RW, Dwosh I, Lindsey S, Pincus T, Wolfe F. The American College of Rheumatology 1991 revised criteria for the classification of global functional status in rheumatoid arthritis. Arthritis Rheum. 1992;35:498-502.

15. Steinbrocker $\mathrm{O}$, Traeger $\mathrm{CH}$, Batterman RC. Therapeutic criteria in rheumatoid arthritis. J Am Med Assoc. 1949;140:659-62.

16. Holmes TH, Rahe RH. The Social Readjustment Rating Scale. J Psychosom Res. 1967;11:213-8.

17. Masuda M, Holmes TH. The Social Readjustment Rating Scale: a cross-cultural study of Japanese and Americans. J Psychosom Res. 1967;11:227-37.

18. Morimoto K. Sutoresu-kiki no Yobou-igaku (Preventive medicine for stress-crisis). Tokyo: NHK Publishing; 1997.

19. Nagano J, Sudo N. Development of a self-administered questionnaire to assess disease-prone personalities: Item construction and content validity. Kenko Kagaku. 2001;23:41-52. English translation: http://hdl.handle.net/2324/724.

20. Nagano J, Sudo N, Kaihara C, Shimura M, Kubo C. Validity and reliability of the Stress Inventory: self-administered questionnaire to assess disease-prone personalities. Kenko Shien. 2001;3:107-19. Enlish translation: http:// hdl.handle.net/2324/15513.

21. Straub RH, Cutolo M. Does stress influence the course of rheumatic diseases? Clin Exp Rheumatol. 2006:24:225-8.

22. Pincus T, Sokka T. Complexities in the quantitative assessment of patients with rheumatic diseases in clinical trials and clinical care. Clin Exp Rheumatol. 2005;23:S1-9.

23. Sifneos PE. Prevalence of Alexithymic Characteristics in Psychosomatic Patients. Psychother Psychosom. 1973;22:255-62.

24. Taylor GJ, Bagby RM. New trends in alexithymia research. Psychother Psychosom. 2004;73:68-77.

25. Kojima M. Alexithymia as a prognostic risk factor for health problems: a brief review of epidemiological studies. BioPsychoSocial Medicine. 2012;6:21.

26. Kano M, Fukudo S. The alexithymic brain: the neural pathways linking alexithymia to physical disorders. BioPsychoSocial Medicine. 2013;7:1.

27. Bagby RM, Parker JD, Taylor GJ. The twenty-item Toronto Alexithymia Scale-I. Item selection and cross-validation of the factor structure. J Psychosomatic Res. 1994;38:23-32.

28. Lane RD, Quinlan DM, Schwartz GE, Walker PA, Zeitlin SB. The Levels of Emotional Awareness Scale: a cognitive-developmental measure of emotion. J Pers Assess. 1990;55:124-34.

29. Moriguchi Y, Komaki G. Neuroimaging studies of alexithymia: physical, affective, and social perspectives. BioPsychoSocial Medicine. 2013;7:8. 